\title{
Supermodes of High-Repetition-Rate Passively Mode-Locked Semiconductor Lasers
}

\author{
Randal A. Salvatore, Steve Sanders, Thomas Schrans, and Amnon Yariv, Life Fellow, IEEE
}

\begin{abstract}
We present a steady-state analysis of high-repetitionrate passively mode-locked semiconductor lasers. The analysis includes effects of amplitude-to-phase coupling in both gain and absorber sections. A many-mode eigenvalue approach is presented to obtain supermode solutions. Using a nearest-neighbor mode coupling approximation, chirp-free pulse generation and electrically chirp-controlled operation are explained for the first time. The presence of a nonzero alpha parameter is found to change the symmetry of the supermode and significantly reduce the mode-locking range over which the lowest order supermode remains the minimum gain solution. An increase in absorber strength tends to lead to downchirped pulses. The effects of individual laser parameters are considered, and agreement with recent experimental results is discussed.
\end{abstract}

\section{INTRODUCTION}

$\mathbf{P}$ REVIOUSLY, the theory of passive modelocking has been analyzed thoroughly in the time domain [1]. Haus' analysis has provided a clear picture of the evolution of pulses through gain, absorptive, and bandwidth-limiting elements within a cavity. A steady-state solution was found when these effects are included. Certain approximations were deemed necessary in order to present an analytic solution. For example, in the steady-state solution, a symmetric and unchirped pulse envelope is assumed as limited by the approximation of all time-domain effects only up to the quadratic term. The model has been extended to include chirped pulses due to selfphase modulation (SPM) yet only for a fast absorber [2], [3], and still restricts the analysis to exponents quadratic in time and achieves symmetric pulses. No recovery is assumed to occur during pulses. Additionally, both models include an approximation of the discrete-mode spectrum by a continuous spectrum. Although the latter approximation works well for mode-locked lasers having many closely-spaced modes, and a slightly-varying gain with frequency, it, along with the assumption of no material recovery during the pulse, is not adequate for the case of high-repetition-rate passively modelocked lasers $(\geq 50 \mathrm{GHz})$. In this case, the difference in gain

Manuscript received August 1, 1995; revised January 30, 1996. This work was supported by the National Science Foundation under Grant ECS-9001272 and by ARPA and the Office of Naval Research under Grant N00014-91-J1195.

R A. Salvatore is with the Electrical and Computer Engineering Department, University of California, Santa Barbara, Santa Barbara, CA 93106 USA. S. Sanders is with SDL Inc., 80 Rose Orchard Way, San Jose, CA 95134 USA.

T. Schrans is with the Ortel Corp., 2015 West Chestnut Street, Alhambra, CA 91803 USA.

A. Yariv is with the Department of Applied Physics 128-95, California Institute of Technology, Pasadena, CA 91125 USA.

Publisher Item Identifier S 0018-9197(96)04131-0. between neighboring modes can be significant, and typically only a small number of modes (around 3-10) dominate.

Active modelocking, on the other hand, has been analyzed thoroughly in both the time domain and the frequency domain [4]-[6]. It has been suggested that passive mode-locking should be analyzed in the time domain since simple products in the time-domain analysis result in cumbersome convolutions in the frequency domain analysis [7], however, in the case of high-repetition-rate passive modelocking, where few modes are involved and the induced carrier modulation is much closer to a sinusoid [8], the frequency domain approach becomes more appropriate. In this paper, we present a steadystate analysis of passive modelocking directed toward highrepetition-rate semiconductor lasers. The analysis is done in the frequency domain extending that presented in [8]. For the first time, passive mode-locking supermodes are found while amplitude-to-phase coupling from slow saturation is permitted. Section II describes the model and arrives at an equation for each mode in the supermode. It incorporates dispersive effects through the common semiconductor laser parameters and unlike previous frequency domain calculations, does not force all modes beyond (the minimum) three modes to contribute zero coupling. Section III describes the eigenvalue formulation used to arrive at a self-consistent solution of the coupled nonlinear equations. Section IV presents an approximate analytical expression based on (the minimum) three modes in order to reduce the complexity and allow one to build physical intuition about the gain requirements and amplitudes and phases of the supermode spectrum. Section $\mathrm{V}$ presents results for the full calculation. Section VI compares the results with experiments for high-repetition-rate passively mode-locked lasers. Finally, Section VII includes conclusions.

\section{THE MODEL}

High-repetition-rate. modelocking $(\geq 50 \mathrm{GHz})$ was first demonstrated by Vasil'ev [9] and by Sanders et al. [10]. To date, semiconductor lasers are the only mode-locked lasers that have been able to generate repetition rates of hundreds of GHz. Due to their large material gain coefficients, fast recovery times, and the ability to be made into short monolithic cavities, high-repetition-rate pulse trains can be generated easily. Typically, high-repetition-rate lasers involve a monolithic semiconductor laser structure, meaning no external cavity is used. The model presented is intended to analyze the monolithic multisection laser, and no intention of including an external cavity is made here although one could easily modify 


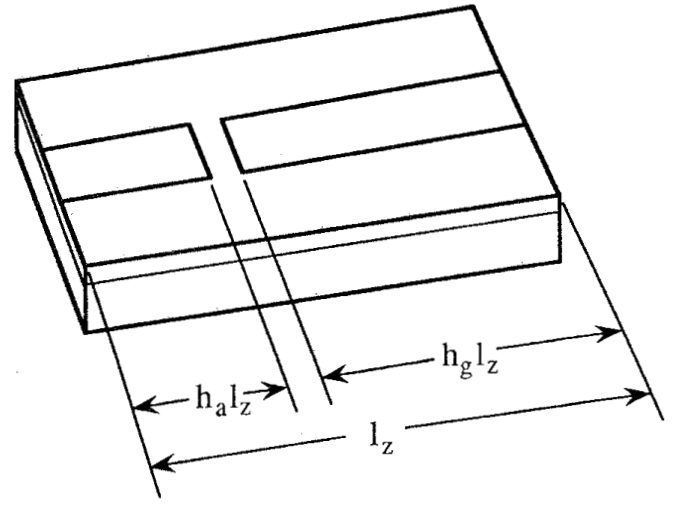

Fig. 1. Schematic for two-section monolithic passively mode-locked laser.

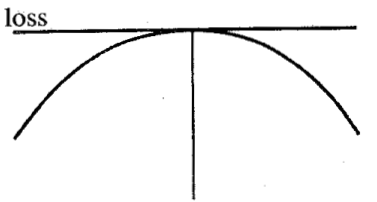

Single Mode

(a)

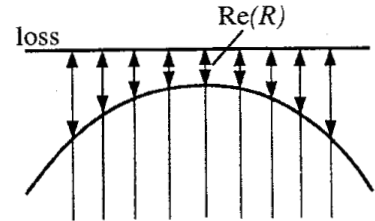

AM Mode-locking

(b)
Fig. 2. Without any mode coipling, a homogeneously broadened laser will lase in the single mode at which the gain and loss are (a) equal. Allowing mode coupling, amplitude modulated (AM) passive modelocking may permit a cooperative saturation of the absorber during some part of the repetition cycle and allow supermode lasing to occur (b) with a lower threshold gain than if mode coupling were disallowed.

the modeled cavity to include a reflection-free facet and some length of free space to account for an external cavity.

Passive modelocking requires a minimum of two sections such that one section is pumped above transparency and one remains below. A standard two-section monolithic passively mode-locked laser structure is shown in Fig. 1. More complex structures have been made to achieve Bragg filtering [11], incorporate additional sections [12], [13], change recombination rates [14], or develop transient gratings to increase the effectiveness of absorber saturation [12]. The steady-state effects of each of these can be taken into account by adjusting the appropriate parameters of the model in Fig. 1.

Physically, one may model the average net gain of a semiconductor laser as having an approximately parabolic spectrum near its peak. Typically, if one pumps the gain strongly enough, it will reach a point at which the gain equals the loss as shown in the left side of Fig. 2. If the gain equals the loss for some mode, this mode will start lasing, the homogeneous gain will become clamped, and further pumping will go into generating light in the lasing mode.

On the other hand, if one allows a coupling to exist between the modes, the presence of light in mode $n$, under some conditions, can make it easier for the light in mode $n+1$ (and vice versa) to saturate through the absorber at certain times during the repetition cycle. Thus, the laser may lase at a lower average carrier density than it could if mode coupling were disallowed [see Fig. 2(b)]. One can write an equation for the net gain of each mode including the coupling effects due to each of its neighboring modes. Also there are phase effects, and for stable mode-locking one requires that all the modes will be equally spaced in frequency. The rest of this section will be devoted to deriving an equation for each of these coupled modes which will subsequently be solved to find the supermode for the high-repetition-rate passively mode-locked laser.

The net optical field inside the laser can be written as a sum over individual modes,

$$
\vec{E}(\vec{r}, t)=\sum_{n} \mathcal{E}_{n}(t) \vec{u}_{n}(\vec{r})
$$

where $\mathcal{E}_{n}(t)$ represents the time dependence of mode $n$, and $\vec{u}_{n}(\vec{r})$ represents the $n$th spatial eigenmode of the cold cavity and satisfies $\nabla^{2} \vec{u}_{n}(\vec{r})+\mu_{0} \varepsilon_{n} \Omega_{n}^{2} \vec{u}_{n}(\vec{r})=0$. Here, $\mu_{0}$ is the magnetic permeability of free space, $\varepsilon_{n}$ is the electric permittivity, and $\Omega_{n}$ is the resonant frequency of the $n$th mode of the cold cavity. Assuming we have some uniform guiding (through index or gain-guiding) structure longitudinally throughout the laser, we can write

$$
\vec{u}_{n}(\vec{r})=\sqrt{2} \vec{a}(x, y) \cos \left(\beta_{n} z\right) .
$$

These modes of the cold cavity may be delta-function normalized,

$$
\int \vec{u}_{n}(\vec{r}) \cdot \vec{u}_{m}(\vec{r}) d V=V_{c} \delta_{n m}
$$

Similar to (1), the net electronic polarization can be written as a sum of terms separable in space and time. Upon writing the wave equation for the net field and polarization and projecting onto $\vec{u}_{n}(\vec{r})$ :

$$
\begin{aligned}
& \frac{d^{2} \mathcal{E}_{n}(t)}{d t^{2}}+\frac{1}{\tau_{p n}} \frac{d \mathcal{E}_{n}(t)}{d t}+\Omega_{n}^{2} \mathcal{E}_{n}(t) \\
& =\frac{-1}{\varepsilon_{n}} \frac{d^{2} \mathcal{P}_{n}(t)}{d t^{2}}
\end{aligned}
$$

where $\mathcal{P}_{n}(t)=\left(1 / V_{c}\right) \int \vec{P}(\vec{r}, t) \cdot \vec{u}_{n}(\vec{r}) d V$ is the projection of the polarization on mode $n$. Here $\tau_{p n}$ represents the photon lifetime for the $n$th mode.

With the optical frequency much greater than the repetition rate, we may write $\tilde{E}_{n}(t)$ as the slowly varying complex envelope of $\mathcal{E}_{n}(t)$ such that

$$
\mathcal{E}_{n}(t)=\frac{1}{2} \tilde{E}_{n}^{\prime}(t) e^{i w_{n} t}+\text { c.c. },
$$

where $\omega_{n}$ is the optical angular frequency of the $n$th lasing mode ( $\omega_{n} \neq \Omega_{n}$ for nonzero detuning), and correspondingly $\tilde{P}_{n}(t)$ may be written as the slowly varying complex envelope of the polarization. Thus,

$$
\begin{aligned}
& \frac{d \tilde{E}_{n}(t)}{d t}-i\left(\Omega_{n}-\omega_{n}\right) \tilde{E}_{n}(t)+\frac{1}{2 \tau_{p n}} \tilde{E}_{n}(t) \\
& =-\frac{i \omega_{n}}{2 \varepsilon_{n}} \tilde{P}_{n}(t)
\end{aligned}
$$


where $\tilde{P}_{n}(t)$ will contain coupling terms to electric fields spaced at harmonics of the repetition rate, $\Delta=\omega_{n}-\omega_{n-1}$, since the net polarization is given by

$$
\vec{P}(\vec{r}, t)=\varepsilon_{0} \chi(\vec{r}, t) \vec{E}(\vec{r}, t)
$$

where

$$
\begin{gathered}
\chi(\vec{r}, t)=f\left(\omega_{n}\right) \chi_{0}(\vec{r})+\sum_{k>0} f\left(\omega_{n+k}\right) \chi_{k}(\vec{r}) \\
\cdot \cos \left[k \Delta t+\psi_{k}(\vec{r})\right]
\end{gathered}
$$

may possess optical-pulse-induced oscillations in the carrier density [8], and $f\left(\omega_{n}\right)$ takes into account the frequency dependent gain or loss of the material. Although in general the material's loss spectrum has somewhat different center and shape than that of the material's gain spectrum, we shall not attempt to model that in this paper.

Because lasers tend to operate at their gain peak and semiconductor lasers have a significant contribution of gaindependent phase shift at their gain peak, $\chi_{k}(\vec{r})=\chi_{k}^{\prime}(\vec{r})+$ $i \chi_{k}^{\prime \prime}(\vec{r})$ presents not only a gain, but a change in refractive index as well. The mode-locked laser is in fact no better in this respect. It tends to operate at an even longer wavelength than a continuous wave ( $\mathrm{CW}$ ) laser (due to the presence of the absorber) [8] and is expected to produce even a slightly larger amplitude-to-phase coupling factor $\alpha$ in its gain section [15], where $\alpha=-\chi_{k}^{\prime}(\vec{r}) / \chi_{k}^{\prime \prime}(\vec{r})$.

Since $\tilde{P}_{n}(t)$ is computed from a projection of $\vec{P}(\vec{r}, t)$ onto $\vec{u}_{n}(\vec{r})$ over the whole length of the laser, there is a contribution from both the gain and absorbing sections

$$
\begin{aligned}
\tilde{P}_{n}(t)= & f\left(\omega_{n}\right) \frac{\varepsilon_{0}}{V_{c}} \xi_{0} \tilde{E}_{n}(t) \\
& +\frac{\varepsilon_{0}}{V_{c}} \sum_{k>0}\left[f\left(\omega_{n-k}\right)\left(\xi_{k g-} e^{i \psi_{k g}}+\xi_{k a-} e^{i \psi_{k a}}\right)\right. \\
& \cdot \tilde{E}_{n-k}(t)+f\left(\omega_{n+k}\right)\left(\xi_{k g+} e^{-i \psi_{k g}}+\xi_{k a+} e^{-i \psi_{k a}}\right) \\
& \left.\cdot \tilde{E}_{n+k}(t)\right]
\end{aligned}
$$

where

$$
\xi_{0}=\int \chi_{0}(\vec{r})\left|\vec{u}_{n}(\vec{r})\right|^{2} d V
$$

and

$$
\xi_{k(g / a) \pm} e^{i \psi_{k(g / a)}}=\int \chi_{k(g / a)}(\vec{r}) \vec{u}_{n \pm k}(\vec{r}) \cdot \vec{u}_{n}(\vec{r}) d V
$$

(The notation $(g / a)$ indicates quantities pertaining to the gain or absorber region, respectively.) We will use $\chi^{\prime \prime}=\hat{g} \mu_{r} c / \omega_{0}$ to relate the material gain coefficient, $\hat{g}$, to the imaginary part of the susceptibility with $\mu_{r}$ being the cold cavity refractive index.

The imaginary part of (10) yields

$$
f\left(\omega_{n}\right) \frac{\xi_{0}^{\prime \prime}}{V_{c}}=\frac{f\left(\omega_{n}\right) \Gamma \mu_{r} c}{\omega_{0}}\left[\hat{g} h_{g}+\hat{a} h_{a}\right]
$$

and this term is proportional to the average single pass gain where $\Gamma$ is the confinement factor, $c$ is the speed of light, $\hat{a}$ is the material gain coefficient of the absorber section $(\hat{a}<$ $0), l_{z}$ is the total laser length, and $h_{g}$ and $h_{a}$ are the ratios of the gain section and absorber section lengths, respectively, to the full laser length. The term $\xi_{k g \pm z} e^{-i \psi_{k g}}$ will be determined from the carrier dynamics by using a linear approximation for the change in optical gain (loss) versus carrier density for the gain (absorber) section with $\hat{g}=G\left[n_{g}(t)-n_{0}\right]$. Here, $G$ is the differential gain, $n_{g}(t)$ is the time dependent carrier density, and $n_{0}$ is the carrier density at transparency. Correspondingly, $\hat{a}=A\left[n_{a}(t)-n_{0}\right]$ for the absorber.

Gain and absorber dynamics result from the photon intensity, which is proportional to

$$
S(z, t)=s_{0}+\sum_{k} s_{k}(z) \cos (k \Delta t)
$$

with

$$
s_{0}=\left\langle s_{0}(z)\right\rangle_{\lambda}=\frac{1}{2}\left\langle\sum_{n=-q}^{q}\left|\tilde{E}_{n}(t)\right|^{2} u_{n}^{2}(z)\right\rangle_{\lambda}
$$

and

$$
\begin{aligned}
s_{k}(z) & =s_{k} \cos \left(\frac{k \pi z}{l_{z}}\right) \\
& =\left\langle\sum_{n=k-q}^{q} \tilde{E}_{n}(t) \tilde{E}_{n-k}^{*}(t) u_{n}(z) u_{n-k}(z)\right\rangle_{\lambda},
\end{aligned}
$$

where \langle\rangle$_{\lambda}$ represents a spatial average over a wavelength. Permitting this form, one notices from the carrier rate equation that a modulation in the light intensity will induce a modulation in the carrier density at the same fundamental and harmonics of that frequency. However, the effect of both the small number of modes and the shorter in-phase overlaps of quickly beating pairs of modes causes the coupling of higher harmonics to drop off. Ignoring the terms responsible for second nearest neighbor and higher coupling terms to simplify the problem and still keep it suitable for high-repetition rate modelocking, from the carrier rate equation,

$$
\begin{aligned}
\frac{d n_{(g / a)}(z, t)}{d t}= & R_{p(g / a)}-(\hat{g} / \hat{a})(z, t) S(z, t) \\
& -\frac{n_{(g / a)}(z, t)}{\tau_{(g / a)}},
\end{aligned}
$$

we find a saturated material gain $\hat{g}$ for the gain section, dependent on the gain recombination time $\tau_{g}$ and the injection pumping $R_{p(g / a)}$ and correspondingly $\hat{a}$ for the absorber section dependent on the absorber recombination time $\tau_{a}$,

$$
\begin{aligned}
& \hat{g}=\frac{\hat{g}^{\prime}}{1+G \tau_{g} s_{0}} \\
& \hat{a}=\frac{\hat{a}^{\prime}}{1+A \tau_{a} s_{0}} .
\end{aligned}
$$

Here, $\hat{g}^{\prime}$ and $\hat{a}^{\prime}$ represent the unsaturated gain and unsaturated loss. Additionally, the carrier density is written $n_{(g / a)}(z, t)=$ 
$n_{0(g / a)}+n_{1(g / a)}(z) \cos \left[\Delta t+\psi_{(g / a)}\right]+\cdots$, and terms showing modulation at the first harmonic in the rate equation lead to:

$$
\begin{aligned}
\tilde{n}_{g 1}(z) & =\frac{-\hat{g} s_{1}(z)}{\sqrt{\Delta^{2}+\left(\frac{1}{\tau_{g}}+G s_{0}\right)^{2}}}, \\
\psi_{g} & =-\arctan \left(\frac{\Delta}{\frac{1}{\tau_{g}}+G s_{0}}\right) \\
\tilde{n}_{a 1}(z) & =\frac{-\hat{a} s_{1}(z)}{\sqrt{\Delta^{2}+\left(\frac{1}{\tau_{a}}+A s_{0}\right)^{2}}},
\end{aligned}
$$

and

$$
\psi_{a}=-\arctan \left(\frac{\Delta}{\frac{1}{\tau_{a}}+A s_{0}}\right) .
$$

So the carrier modulation becomes small and it lags the optical pulses by nearly $\pi / 2$ radians since the repetition rate is well beyond the recombination rate or saturation rate. Computing the spatial integrals in (11), we find that

$$
\frac{\xi_{1 g \pm} e^{i \psi_{g}}}{V_{c}}=\left(-\alpha_{g}+i\right) \frac{\mu_{r}^{2}}{\omega_{0} \tau_{p}} \kappa_{g} s_{1} e^{i \psi_{g}}
$$

where

$$
\begin{aligned}
\kappa_{g}= & \frac{-G \tilde{g}_{0}}{\sqrt{\Delta^{2}+\left(\frac{1}{\tau_{g}}+G s_{0}\right)^{2}}} \\
& \cdot\left[\frac{1}{2}+\frac{1}{4 \pi h_{g}} \sin \left(2 \pi h_{g}\right)\right]
\end{aligned}
$$

and

$$
\tilde{g}_{0}=\Gamma \hat{g} h_{g} \frac{c \tau_{p}}{\mu_{r}}
$$

is the normalized gain. Likewise for the part of the integral over the absorber,

$$
\frac{\xi_{1 a \pm} e^{i \psi_{a}}}{V_{c}}=\left(-\alpha_{a}+i\right) \frac{\mu_{r}^{2}}{\omega_{0} \tau_{p}} \kappa_{a} s_{1} e^{i \psi_{a}}
$$

where

$$
\begin{aligned}
\kappa_{a}= & \frac{-A \tilde{a}_{0}}{\sqrt{\Delta^{2}+\left(\frac{1}{\tau_{a}}+A s_{0}\right)^{2}}} \\
& \cdot\left[\frac{1}{2}+\frac{1}{4 \pi h_{a}} \sin \left(2 \pi h_{a}\right)\right]
\end{aligned}
$$

and

$$
\tilde{a}_{0}=\Gamma \hat{a} h_{a} \frac{c \tau_{p}}{\mu_{r}} .
$$

One can write the single pass gain from (12) along with its corresponding phase contribution. Also, for generality, one should allow the inclusion of a frequency dependence [16] of $\alpha$ [accomplished through $\alpha_{(g / a) n}$ ], giving the single pass net gain and phase effects that are not due to coupling as

$$
\frac{\xi_{0 n}}{V_{c}}=f\left(\omega_{n}\right) \frac{\mu_{r}^{2}}{\omega_{0} \tau_{p}}\left[\left(-\alpha_{g n}+i\right) \tilde{g}_{0}+\left(-\alpha_{a n}+i\right) \tilde{a}_{0}\right]
$$

For steady state, we can ignore all time derivatives and using (6), (9), (23), (25), (26), (28), and (29), the equation for mode $n$ becomes,

$$
\begin{aligned}
& \left\{2 i \tau_{p n}\left(\Omega_{n}-\omega_{n}\right)-1+f\left(\omega_{n}\right)\left[\left(1+i \alpha_{g n}\right) \tilde{g}_{0}\right.\right. \\
& \left.\left.\quad+\left(1+i \alpha_{a n}\right) \tilde{a}_{0}\right]\right\} \tilde{E}_{n} \\
& \quad+\frac{s_{1}}{s_{0}}\left(\tilde{\eta}_{n-} \tilde{E}_{n-1}+\tilde{\eta}_{n+} \tilde{E}_{n+1}\right)=0
\end{aligned}
$$

where we have defined coupling coefficients for the nearest neighbor modes,

$$
\begin{aligned}
\tilde{\eta}_{n-}= & f\left(\omega_{n}\right)\left[\kappa_{g}\left(1+i \alpha_{g n}\right) e^{i \psi_{g}}\right. \\
& \left.+\kappa_{a}\left(1+i \alpha_{a n}\right) e^{i \psi_{a}}\right] \frac{s_{0}}{2} \\
\tilde{\eta}_{n+}= & f\left(\omega_{n}\right)\left[\kappa_{g}\left(1+i \alpha_{g n}\right) e^{-i \psi_{g}}\right. \\
& \left.+\kappa_{a}\left(1+i \alpha_{a n}\right) e^{-i \psi_{a}}\right] \frac{s_{0}}{2} .
\end{aligned}
$$

These two terms are completely determined by the structure of the laser and the average photon intensity.

Let a single detuning in the separation of modes be defined, $\delta=\omega_{n}-\Omega_{n}-\left(\omega_{n-1}-\Omega_{n-1}\right)$, since for stable modelocking the detuning of the repetition rate, $\delta$, must equal the detuning in the separation between all neighboring modes. The detuning, $\delta_{n}$, of mode $n$ with respect to $\Omega_{n}$ is then the detuning of the zeroth mode plus $n$ times the repetition rate detuning, $\delta_{n}=\delta_{0}+n \delta$. The general equation then for the $n$th mode with nearest neighbor coupling, for $\alpha$ parameters incorporated for the gain and absorber, and with geometric overlap factors included is

$$
\begin{aligned}
& {\left[-2 i \tau_{p n}\left(\delta_{0}+n \delta\right)+\left(1+i \alpha_{g n}\right) \tilde{g}_{n}+\left(1+i \alpha_{a n}\right) \tilde{a}_{n}-1\right]} \\
& \cdot \tilde{E}_{n}+\tilde{s}_{1}\left(\tilde{\eta}_{n-}-\tilde{E}_{n-1}+\tilde{\eta}_{n+} \tilde{E}_{n+1}\right)=0 .
\end{aligned}
$$

Here $\tilde{s}_{1}=s_{1} / s_{0}$, and the material gain bandwidth is taken into account with $\tilde{g}_{n}=f\left(\omega_{n}\right) \tilde{g}_{0}$, and $\tilde{a}_{n}=f\left(\omega_{n}\right) \tilde{a}_{0}$.

\section{THE SOLUTION}

The coupled nonlinear equations (33) can be solved systematically. Also, one should solve the problem for a large enough number of equations such that the result does not depend strongly on the fact that the modes beyond those considered have been forced to have an electric field of zero. To reduce the number of parameters for the calculation, it will be helpful to transform to dimensionless parameters,

$$
\begin{aligned}
s & =\frac{A}{G}, \\
r & =\frac{\tau_{a}}{\tau_{g}}, \\
\tilde{\Delta} & =\Delta \tau_{g}, \\
\tilde{s}_{0} & =G \tau_{g} s_{0}, \\
\tilde{\kappa}_{g} & =\frac{\kappa_{g}}{G \tau_{g}},
\end{aligned}
$$

and

$$
\tilde{\kappa}_{a}=\frac{\kappa_{a}}{A \tau_{a}} .
$$


One may subtract out the detuning of mode zero from the set of equations (33). Defining a constant,

$$
R=\left(\tilde{\eta}_{0-} \tilde{E}_{-1}+\tilde{\eta}_{0+} \tilde{E}_{+1}\right) \frac{\tilde{s}_{1}}{\tilde{E}_{0}}
$$

$\operatorname{Im}(R)$ is the component of detuning of the center mode due to mode coupling and $\operatorname{Re}(R)$ is the reduction in required average gain for the center mode due to mode coupling, similar to that discussed in [17]. Taking the imaginary part of the $n=0$ equation and subtracting it from the general mode $n$ equation leads to

$$
\begin{aligned}
& {\left[-2 i \tau_{p_{n}} n \delta+\left(1+i \alpha_{g n}\right) \tilde{g}_{n}+\left(1+i \alpha_{a n}\right) \tilde{a}_{n}\right.} \\
& \left.\quad-i\left(\alpha_{g 0} \tilde{g}_{0}+\alpha_{a 0} \tilde{a}_{0}\right)-i \operatorname{Im}(R)-1\right] \tilde{E}_{n} \\
& \quad+\tilde{s}_{1}\left(\tilde{\eta}_{n-} \tilde{E}_{n-1}+\tilde{\eta}_{n+} \tilde{E}_{n+1}\right)=0 .
\end{aligned}
$$

The net gain spectrum of the semiconductor material is concave downward and may be represented by the form $f\left(\omega_{n}\right)=$ $1 /\left[1+\left(\omega_{n}-\omega_{0}\right)^{2} /(\Delta \omega)^{2}\right]$. Since to second order, one may write $f\left(\omega_{n}\right)=1-b n^{2}$, substituting this, and since $b \ll 1$ and the coupling term is of the same order, we may ignore their product which goes like $b^{2}$. Now the general equation for mode $n$ with center mode detuning subtracted finally becomes

$$
\begin{aligned}
& {\left[-2 i \tau_{p n} n \delta+\left(\tilde{g}_{0}+\tilde{a}_{0}\right)\left(1-b n^{2}\right)\right.} \\
& \left.\quad-i b n^{2}\left(\alpha_{g n} \tilde{g}_{0}+\alpha_{a n} \tilde{a}_{0}\right)-1-i \operatorname{Im}(R)\right] \tilde{E}_{n} \\
& \quad+\tilde{s}_{1}\left(\tilde{\eta}_{n-} \tilde{E}_{n-1}+\tilde{\eta}_{n+} \tilde{E}_{n+1}\right)=0 .
\end{aligned}
$$

Considering a set of $2 q+1$ modal equations (all are complex except for the $n=0$ equation), there are $4 q+1$ real equations and a list of $4 q+3$ unknowns including $4 q+1$ unknowns to specify the fields [we may take $\arg \left(\tilde{E}_{0}\right)=0$ to define an absolute optical phase $]$ and two other unknowns, $\tilde{g}_{0}$ and $\delta$. The phase of the repetition rate is also a degree of freedom and one may specify $\arg \left(\tilde{s}_{1}\right)=0$. Then, the modulation response of the laser sections can be referenced relative to the phase of the optical pulses. Since physically one considers a laser operating with a specific dc pumping (or more appropriately here, a constant average output) power, one may specify a particular average cavity photon intensity for $\tilde{s}_{0}$. The latter two conditions, without loss of generality, reduce the number of unknowns in the field vector to $4 q-1$, making the problem completely determined. Due to the nonlinear dependence of the parameters $\tilde{g}_{0}, \tilde{a}_{0}, \delta, \operatorname{Im}(R), \hat{\eta}$ 's, and $\tilde{s}_{1}$ on the vector $\overrightarrow{\tilde{E}}$, the problem remains challenging. However, the solution is vastly simplified by viewing it as an eigenvalue problem. For example, one may directly write the problem in a matrix form as (43) found at the bottom of the page. Through multiplications of the rows by the appropriate complex factors

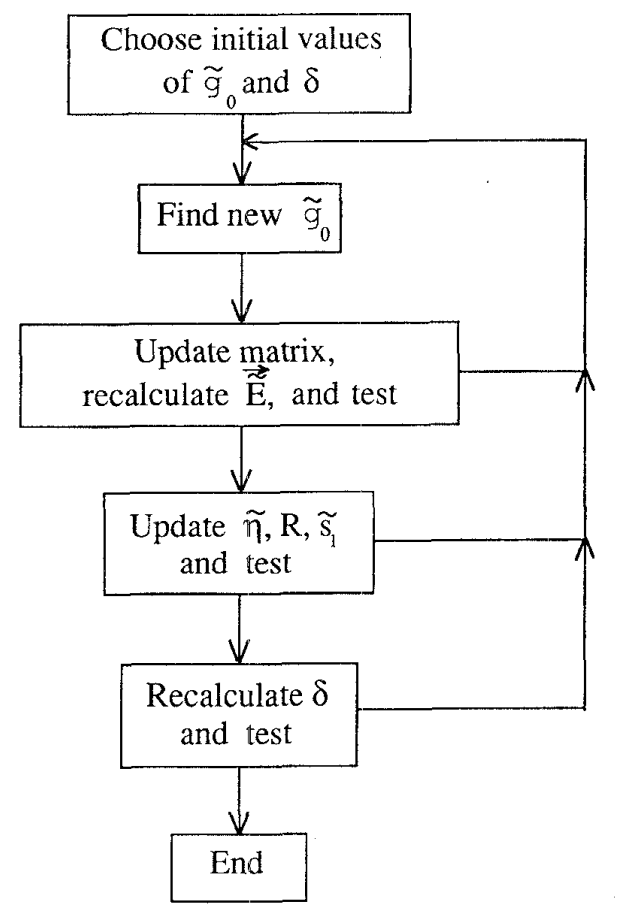

Fig. 3. Flowchart of calculation for self-consistent supermode solution.

one may also show that the problem can always be written, having a single complex eigenvalue, $\tilde{\lambda}$, in the form

$$
\left[\mathbf{A}^{m}\left(\tilde{g}_{0}, \delta, \overrightarrow{\tilde{E}}\right)-\mathbf{I} \tilde{\lambda}\right] \overrightarrow{\vec{E}}^{m}=0,
$$

where $\mathbf{A}^{m}\left(\tilde{g}_{0}, \delta, \overrightarrow{\tilde{E}}\right)$ is a modified complex matrix and $\overrightarrow{\tilde{E}}^{m}$ is a modified eigenvector. The problem is more easily solved by keeping it in the form of (43), however. For a nontrivial eigensolution, we require that the real and imaginary parts of the determinant of the matrix in (43) equal zero. This gives two conditions from which one may find a best estimate for $\tilde{g}_{0}$ and $\delta$, and this was done simply through Newton's method. With this better estimate of the eigenvalue we proceed to update the relevant parameters and find a new estimate of the eigenvalue. The process is repeated as shown in Fig. 3 until convergence is reached. The computation gives the supermode solutions of the high-repetition-rate laser for the chosen average operating power $\tilde{s}_{0}$.

\section{Results From AN APProximate THREE-MODE SOLUTION}

The full numerical solution is complicated, involving a large number of interrelated parameters, and it does not quickly

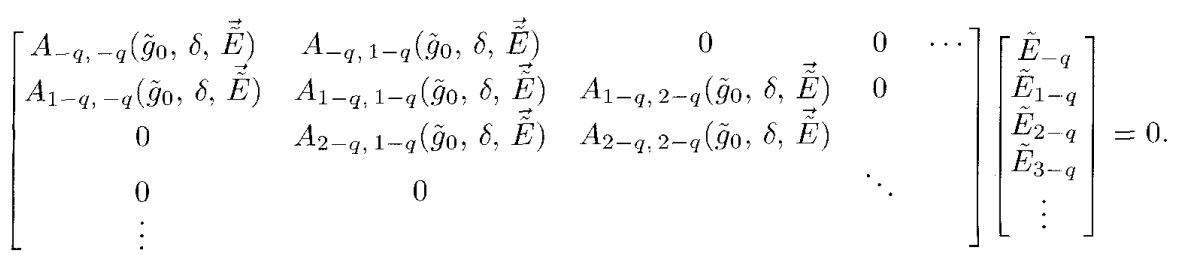


lead to a simple intuitive picture of the effects of the device parameters. To supplement the full numerical solution, an approximate analytical description involving only three modes and an approximation of the supermode symmetry is pursued. One may show that if the $\alpha$ parameters of the gain and absorber sections are ignored, and the gain bandwidth is symmetric relative to the cavity modes, a totally symmetric (odd symmetry) supermode solution for any number of modes will result. The form of the supermode solution will be

$$
\tilde{E}_{n}=\tilde{E}_{-n}^{*}
$$

and one can always find a three-mode solution having all three modes exactly in phase. However, as soon as $\alpha_{g} \neq 0$ or $\alpha_{a} \neq$ 0 is chosen, the symmetry is broken and one finds that now a chirp-free supermode solution of this form will not generally exist.

Thus, no passively mode-locked supermode will exist having the form of (45) when the amplitude-to-phase coupling is taken into account. The relative phases of the modes in the supermode depend strongly on the amplitude-to-phase coupling. One finds, for numerous solutions of the full numerical analysis that once a nonzero $\alpha$ parameter is chosen, the solutions are of the even symmetric form

$$
\tilde{E}_{n} \approx \tilde{E}_{-n}
$$

since phase effects resulting from the $\alpha$ parameter greatly outweigh the effects present when the $\alpha$ 's were zero.

Since a simple, analytic, and reasonably accurate result can be obtained assuming (46) when some nonzero $\alpha$ is present, we derive a solution for three mode-locked modes using this even symmetric assumption. The term $-i b\left[\alpha_{g( \pm 1)} \tilde{g}_{0}+\alpha_{a( \pm 1)} \tilde{a}_{0}\right]$ is found to have little effect on the net gain, amplitudes, or phases of the supermode and will, for this reason, be ignored in this three-mode approximation.

From the $n=1$ and $n=-1$ equations of (42), the expressions

and

$$
\delta=\frac{\operatorname{Im}(R)}{2 \tau_{p} \operatorname{Re}\left(\frac{\tilde{\eta}_{0+}+\tilde{\eta}_{0-}}{\tilde{\eta}_{0+}-\tilde{\eta}_{0-}}\right)},
$$

$$
\frac{\tilde{E}_{1}}{\tilde{E}_{0}}=\frac{i \tilde{s}_{1}\left(\tilde{\eta}_{0+}-\tilde{\eta}_{0-}\right)}{4 \tau_{p} \delta}
$$

can be obtained. Combining this with the $n=0$ equation, we can find the reduction in required gain for the center mode,

$$
\begin{aligned}
\operatorname{Re}(R) & =-\left(\tilde{g}_{0}+\tilde{a}_{0}-1\right) \\
& =-\operatorname{Im}(R) \frac{\operatorname{Im}\left(\tilde{\eta}_{0+}^{2}-\tilde{\eta}_{0-}^{2}\right)}{\operatorname{Re}\left(\tilde{\eta}_{0+}^{2}-\tilde{\eta}_{0-}^{2}\right)} .
\end{aligned}
$$

From (48), we will find that a chirp-free solution will exist if

$$
\tilde{\kappa}_{g} \alpha_{g} \sin \psi_{g}=-\tilde{\kappa}_{a} \alpha_{a} \sin \psi_{a} .
$$

In this case, a soliton-like compensation effect occurs in the monolithic laser cavity. This condition implies that the selfphase modulation (SPM) of the absorber section may exactly oppose the SPM from the gain section [18], [19]. For a larger
TABLE I

Parameter Values used in the Calculations

\begin{tabular}{cccc}
\hline \hline Variable & Symbol & Valuc & Units \\
\hline Number of Modes Considered & $2 q+1$ & 15 & \\
Center Wavelength & $\lambda$ & 0.85 & $\mu \mathrm{m}$ \\
Effective Index of Refraction & $\mu_{r}$ & 3.6 & \\
Differential Gain & $G$ & $1 \times 10^{-15}$ & $\mathrm{~cm}^{2}$ \\
Ratio of Diff. Abs. / Diff. Gain & $s$ & 2.2 & \\
Gain Section Recovery Time & $\tau_{3}$ & $1 \times 10^{-9}$ & $\mathrm{~s}$ \\
Ratio of Abs. Recov. Time / Gain Recov. Time & $r$ & 0.3 & \\
Fundarnertial Reppetition Rate & $\Delta / 2 \pi$ & 80 & $\mathrm{GHz}$ \\
Gain Section $\alpha$ parameter & $\alpha_{g}$ & 4 & \\
Absorber Section $\alpha$ parameter & $\alpha_{a}$ & 2.1 & \\
Pholon Cavity Lifetime & $\tau_{p}$ & 10 & $\mathrm{ps}$ \\
Confinement Factor & $\mathrm{r}$ & 0.05 & \\
Coefficient for $\alpha$ 's dependence on intensity & $\alpha_{l}$ & 0.25 & \\
\hline \hline
\end{tabular}

ratio of $\alpha_{g}: \alpha_{a}$, a net upchirp (optical frequency rising with time during the pulse) due to SPM will occur. In the frequency domain picture this corresponds to a phase term, $e^{i a\left(\omega-\omega_{0}\right)^{2}}$, multiplying the optical spectrum, where $a$ is negative. For a smaller ratio of $\alpha_{g}: \alpha_{a}$, a net downchirp due to SPM is found to occur. A plot of chirp verses the ratio of $\alpha_{g}: \alpha_{a}$ for a specific laser operating point will be shown in the next section, using the full calculation. Evidence of both these regimes has recently been demonstrated [20].

\section{The Full Supermode Calculation}

As formulated in Section III, the high-repetition-rate laser supermode can be found numerically. This may be accomplished even while eliminating all assumptions on the modal phase and removing any restrictions on the number of participating Fabry-Perot modes. One finds that if a large enough number of modes is allowed such that the outermost modes have powers of $<10^{-6}$ compared to the strongest modes, there is little further change in the result if additional modes are included.

Given reasonable parameters for laser material and structure, such as those shown in Table I, one can find the supermode solution. In general, one would not expect the $\alpha$ parameter from the gain and absorber regions to be equal. Previously [15], the dependence of the interband transition component of this parameter has been calculated. One would expect a smaller $\alpha$ parameter for laser sections pumped to lower carrier densities. This, in fact, is found to be an important consideration in finding a stable supermode solution. Lau [8] has calculated supermode solutions for three modes with $\alpha=0$ for both sections. We find reasonably good qualitative agreement with these results even as the number of modes considered is increased. The plots resulting from $\alpha=0$, 


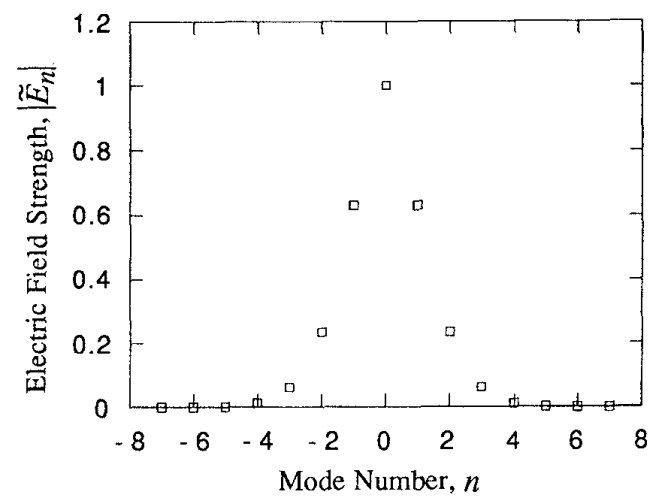

Fig. 4. Calculated mode structure of supermode assuming no amplitude-to-phase coupling, $\alpha_{g}=0, \alpha_{a}=0, \tilde{s}_{0}=2.5$, and using the other parameter values as given in Table I.

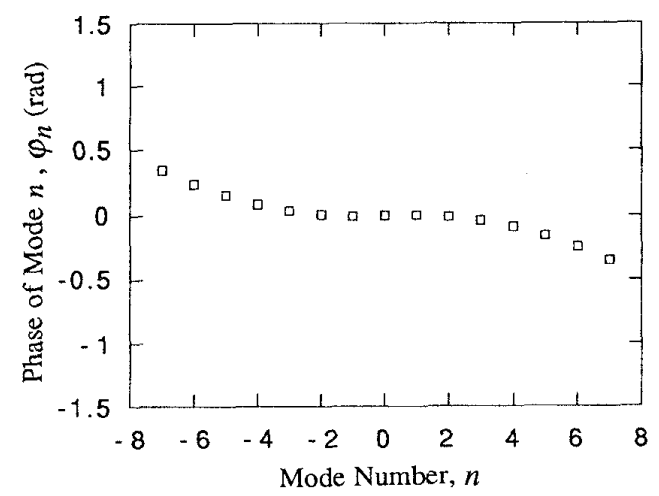

Fig. 5. Calculated phase of the supcrmode assuming no amplitude-to-phase coupling, $\alpha_{2}=0, \alpha_{a}=0, \tilde{s}_{0}=2.5$, and using Table I to define all other parameters.

$\tilde{s}_{0}=2.5$, a 15 -mode calculation, and the parameters in Table I are shown in Figs. 4 and 5. From here on, the frequency dependence of the cold cavity loss is neglected so $\tau_{p n}=\tau_{p}$. Fig. 4 shows the calculated field strengths for the 15-mode supermode. Fig. 5 shows the corresponding modal phases, where $\phi_{n}$ is defined as the optical phase in $\tilde{E}_{n} e^{i\left(w_{n} t+\phi_{n}\right)}$. Clearly, the symmetry of (45) is present here. Fig. 6 shows the threshold gain difference, $\operatorname{Re}(R)$, as a function of $\tilde{s}_{0}$, defined previously and is displayed in units of $10^{-4}$ times the cold cavity loss (from $\tau_{p}$ ). The right side scale of this plot shows the expected detuning, $\delta$, of the cavity repetition rate. Fig. 7 shows the modulation depth at the first harmonic as a function of average intensity. The threshold gain for single mode operation must be greater than the mode-locking threshold gain, meaning $\operatorname{Re}(R)>0$ for stable modelocking to be realized [8]. In ideal amplitude modulated (AM) passive modelocking, a minimum mode coupling is required in order to obtain simultaneous lasing from 3 or more modes of a homogeneously broadened laser. This requires a minimum nonlinearity to be present. Hence, if the average cavity intensity, $\tilde{s}_{0}$, is too low, an inadequate amount of mode coupling is generated, and modelocked operation cannot be obtained. Additionally, if the cavity intensity is large such that the absorber is strongly saturated to

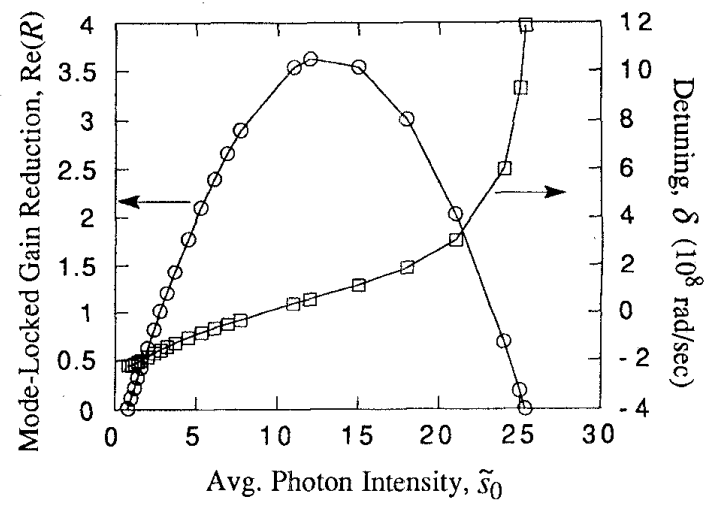

Fig. 6. Calculated plot of $\operatorname{Re}(R)$, the reduction in threshold gain due to mode coupling, versus average cavity intensity is plotted for $\alpha_{g}=0, \alpha_{a}=$ $0, \alpha_{i}=0$. The corresponding detuning, $\delta$, of the repetition rate is shown on the scale at right.

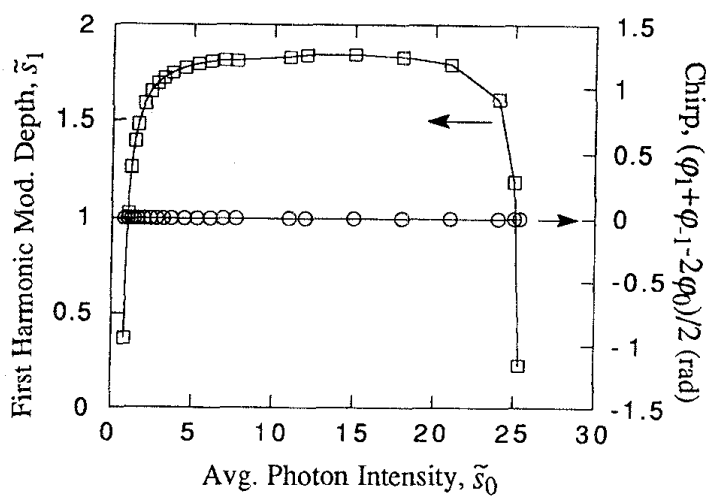

Fig. 7. The calculated modulation depth for the signal at the first harmonic of the repetition rate is plotted for $\alpha_{g}=0, \alpha_{a}=0, \alpha_{I}=0$.

a point far beyond the knee of the nonlinearity, the minimum mode coupling again cannot be obtained. This explains why mode-locking may only be obtained over a finite range in Fig. 6. The right scale in Fig. 7 shows in this case where $\alpha_{g}=$ 0 and $\alpha_{a}=0$, one does not expect SPM to generate any pulse chirp effects and the quadratic phase $\left(\phi_{1}+\phi_{-1}-2 \phi_{0}\right) / 2=$ 0 indicates that, to first order, no linear chirp is present in this case.

As discussed in the previous section, the $\alpha$ parameter can have a large effect on the phase of each optical mode. Assuming an $\alpha$ parameter of $\alpha_{g}=4$ for the gain section, only a limited range of values for $\alpha_{a}$, the $\alpha$ parameter for the absorber section, was found to give stable self-consistent solutions. A calculation of the approximately linear chirp (quadratic phase) at the center of the optical spectrum, $\left(\phi_{1}+\right.$ $\left.\phi_{-1}-2 \phi_{0}\right) / 2$, versus $\alpha_{a} / \alpha_{g}$ is plotted in Fig. 8 for the range of stable mode-locked solutions. The range is quite narrow and corresponds to a region where the SPM effects from absorber and gain nearly cancel as discussed in [20]. The dependence of the $\alpha$ 's on frequency is ignored in this and subsequent plots.

The same plots as shown in Figs. 4-7 can be shown for the case including effects of reasonable nonzero $\alpha$ 's. The new calculated field strength for the 15-mode supermode with 


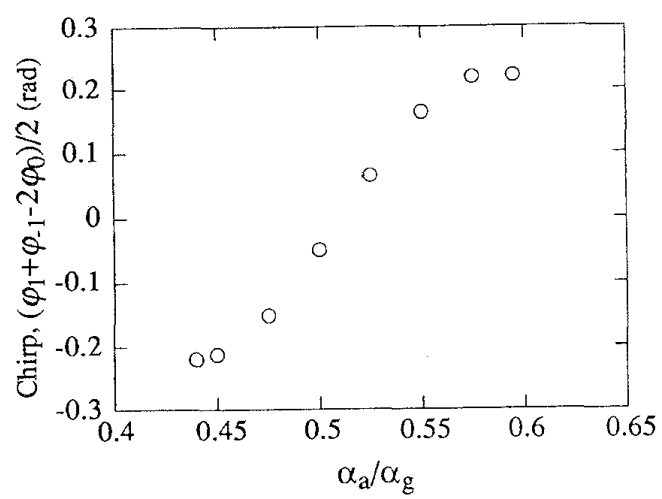

Fig. 8. A calculation of the linear chirp, the quadratic phase around the center of the optical spectrum, for different values of $\alpha_{a} / \alpha_{g}$.

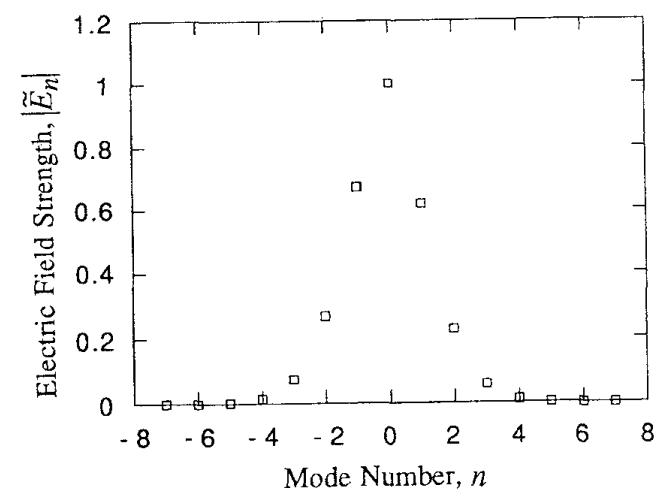

Fig. 9. Calculated mode structure of supermode when allowing amplitude-to-phase coupling, $\alpha_{g}=4, \alpha_{a}=2.1$, and $\tilde{s}_{0}=2.5$. Parameters are exactly as shown in Table $I$

$\alpha_{g}=4$ and $\alpha_{a}=2.1$ is shown in Fig. 9. Fig. 10 shows the corresponding modal phases, $\phi_{n}$. The previously discussed change in supermode symmetry is mainly shown in this plot of $\phi_{n}$. Before discussing the other three plots, it should be mentioned that physically as the gain current in the laser is increased to raise the average intensity, $\tilde{s}_{0}$, one weakens the absorber section through the relation $\tilde{a}_{0}=\tilde{a}_{0}^{\prime} /\left(1+r s \tilde{s}_{0}\right)$, where $\tilde{a}_{0}^{\prime}$ is the section's normalized unsaturated absorption. The strength of the gain is also weakened since we require that $\tilde{g}_{0}+\tilde{a}_{0}-1 \approx 0$. Thus, the two sections both operate closer to transparency as $\tilde{s}_{0}$ is increased. This implies a change in each section's $\alpha$ parameter also occurs and their dependence on $\tilde{s}_{0}$ will be approximated to first order here by $\Delta \alpha_{g}=-\alpha_{l} \Delta \tilde{s}_{0}$ and $\Delta \alpha_{a}=\alpha_{l} \Delta \tilde{s}_{0}$, where $\alpha_{l}$ takes into account a linear decrease (increase) in $\alpha_{g}\left(\alpha_{a}\right)$ as the cavity intensity is increased. Here, $\alpha_{l}$ is taken as 0.25 around the point $\tilde{s}_{0}=2.5$.

Fig. 11 shows the plot of required gain reduction, $\operatorname{Re}(R)$, and the expected detuning in the repetition rate as a function of $\tilde{s}_{0}$. Fig. 12 shows the modulation depth and an estimate of the mode-locked laser's linear chirp $\left(\phi_{1}+\phi_{-1}-2 \phi_{0}\right) / 2$. One can see that the expected mode-locking range over which the coupled equations can be simultaneously satisfied is severely limited when the phase condition including the $\alpha$ parameter is

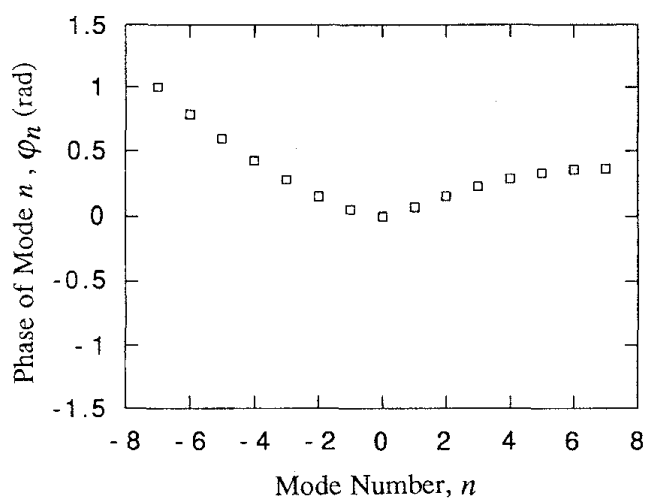

Fig. 10. Calculated phase of supermode when allowing amplitude-to-phase coupling, $\alpha_{g}=4, \alpha_{a}=2.1$. Parameters are exactly as shown in Table 1

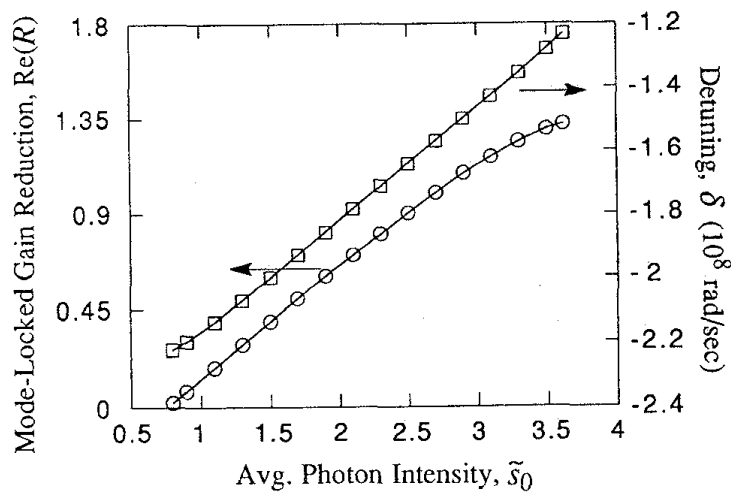

Fig. 11. Calculated plot of $\operatorname{Re}(R)$, the reduction in threshold gain due to mode coupling, versus average cavity intensity is plotted for $\alpha_{g}=4, \alpha_{a}=$ 2.1. The corresponding detuning of the repetition rate is shown on the scale at right.

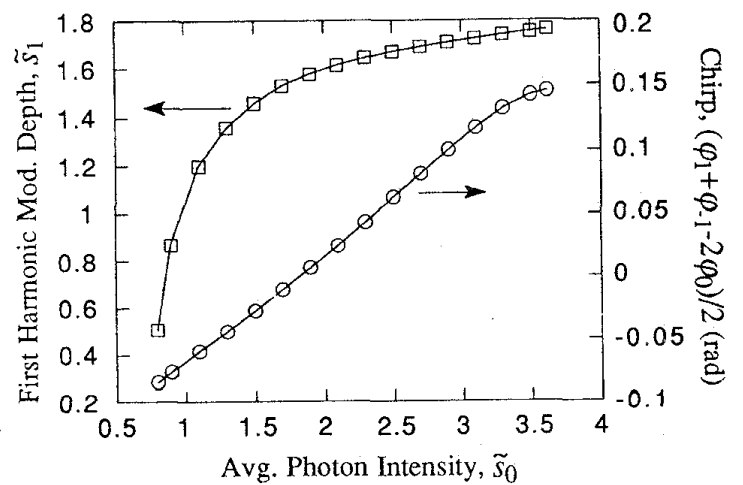

Fig. 12. The calculated modulation depth for the signal at the first harmonic of the repetition rate is plotted for $\alpha_{g}=4, \alpha_{a}=2.1$. The corresponding linear chirp, $\left(\phi_{1}+\phi_{-1}-2 \phi_{0}\right) / 2$ is shown on the scale at right.

considered (although other parameters remain identical). This is a direct result of the presence of the $\alpha$ parameters in the coupling terms and occurs consistently regardless of whether or not one includes more allowed modes in the calculation.

It is expected that the mode-locked laser's operation will change if one modifies the structure or bias parameters. These 


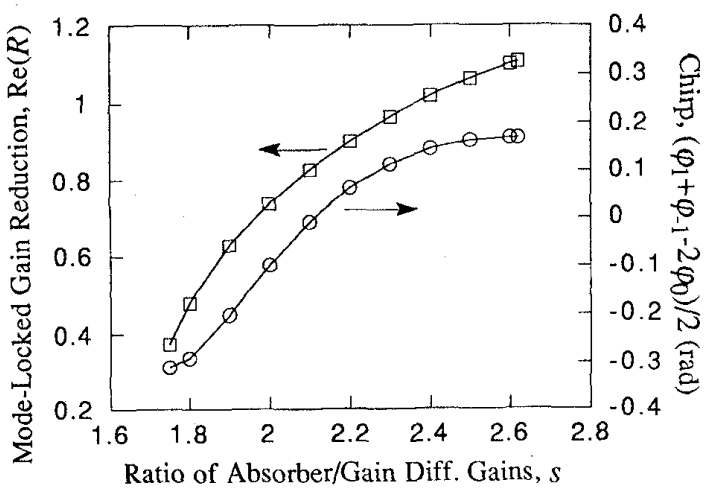

Fig. 13. The calculated variation of $\operatorname{Re}(R)$, the reduction in threshold gain due to mode coupling, plotted against $s$, the ratio of differential absorption to differential gain.

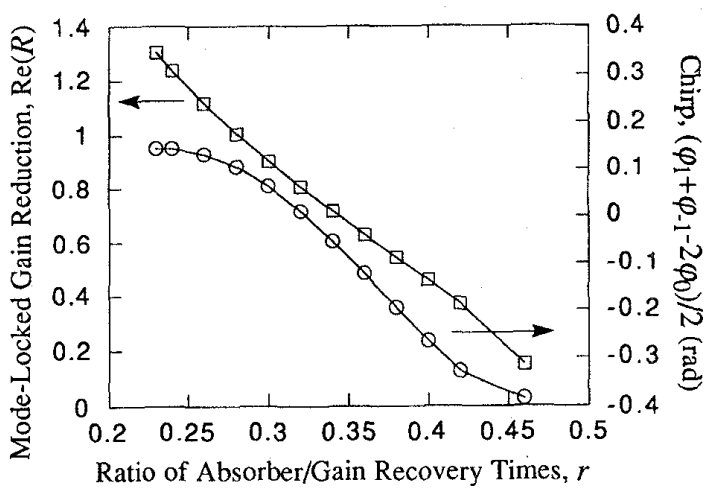

Fig. 14. The calculated variation of $\operatorname{Re}(R)$, the reduction in threshold gain due to mode coupling, plotted against $r$, the ratio of absorber recovery time to gain recovery time.

effects are important if one intends to understand or optimize the laser's operation. We have calculated results one would expect from modifying key laser parameters and using the nearest-neighbor mode coupling approximation for the range of supermode solutions that exist around the case considered in Fig. 9.

One finds that if $s$, the ratio of the differential absorption to differential gain is increased, a larger mode coupling is obtained. This leads to a larger value of $\operatorname{Re}(R)$, the reduction in the mode-locking threshold relative to the single-mode threshold, as shown in Fig. 13, which is expected to lead to a more stable mode-locked supermode. Fig. 13 also shows that a decreased upchirp or increased downchirp is expected to occur if a larger $s$ is present and all other parameters are unchanged.

The effect of $r$, the ratio of absorber recovery time to gain recovery time, is expected to be nearly the opposite. Shown in Fig. 14, an increased $r$ leads to a decrease in the mode-locked gain reduction and ultimately a loss of a stable mode-locked solution altogether as the ratio is increased above $r=0.46$ in this case. Simultaneously the increased value of $r$ will lead to an increased upchirp as shown in Fig. 14. It is known that one can reduce the value of $r$ through stronger reverse bias or ion implantation into the absorber section.

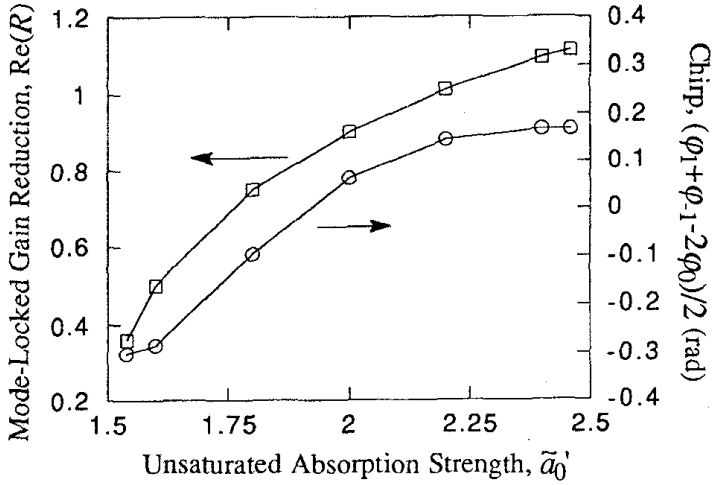

Fig. 15. Calculated value of $\operatorname{Re}(R)$, the reduction in threshold gain due to mode coupling, plotted against $\tilde{a}_{0}^{\prime}$, unsaturated absorption strength.

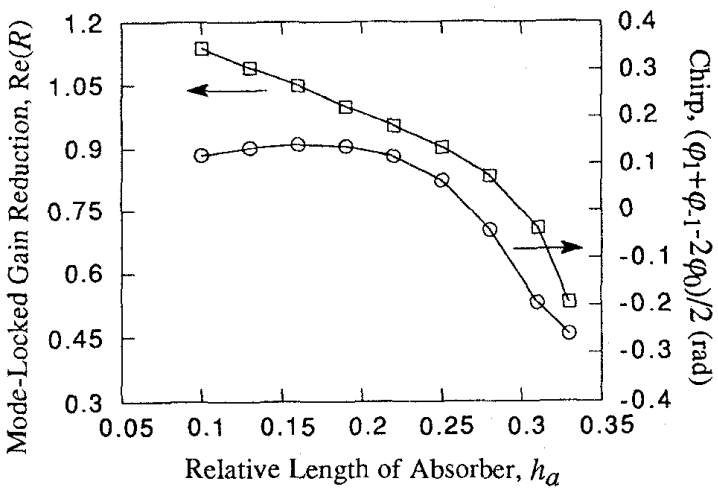

Fig. 16. Calculated value of $\operatorname{Re}(R)$, the reduction in threshold gain due to mode coupling, plotted against $h_{a}$, the physical length of the absorber as a fraction of the laser's full length.

An increase in $\tilde{a}_{0}^{\prime}$, the unsaturated absorption strength of the saturable absorber, is shown to lead (as shown in Fig. 15) to an increase in the mode-locked gain reduction, $\operatorname{Re}(R)$. The strength of the unsaturated absorption is proportional to this section's length and absorption coefficient. An increase in either of these is expected to lead to a more strongly downchirped pulse as shown in Fig. 15. This agrees with expectations described in [20] where a stronger saturable absorber is cited as the reason for a significant downchirp being obtained over most of the experimental chirp-versuscurrent curve.

Unlike the time-domain analyses [1]-[3], the frequency domain analysis allows one to account for the spatial geometry of the laser. Recently, this has been noted by Martins-Filho $e t$ al. [13]. The ratio of the physical length of the absorber to the total laser length, $h_{a}$, is expected to change the effectiveness of mode coupling. If the same absorber strength can be incorporated into a smaller segment of the laser, one can achieve a more effective mode coupling and obtain a larger mode-locked gain reduction, $\operatorname{Re}(R)$. This is consistent with results determined in [21]. Fig. 16 also shows the effects on pulse chirp when the parameter $h_{a}$ is varied.

The mode-locked laser's round-trip frequency is determined by the laser's cavity length. A larger cavity round-trip fre- 


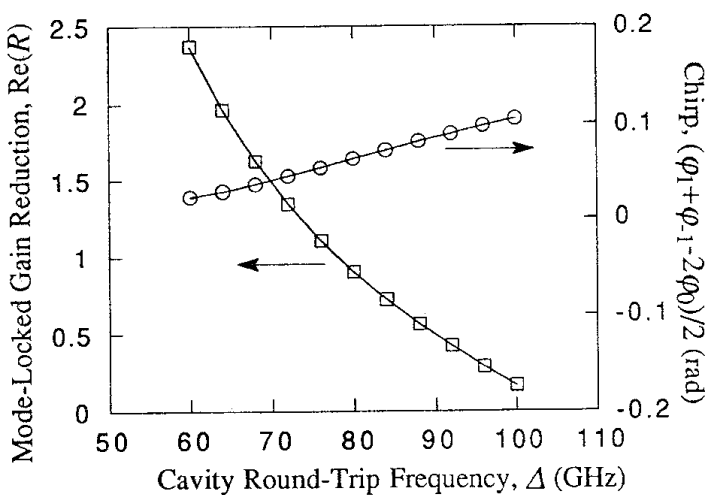

Fig. 17. Calculated value of $\operatorname{Re}(R)$, the reduction in threshold gain due to mode coupling, ploted against $\Delta$, the repetition rate.

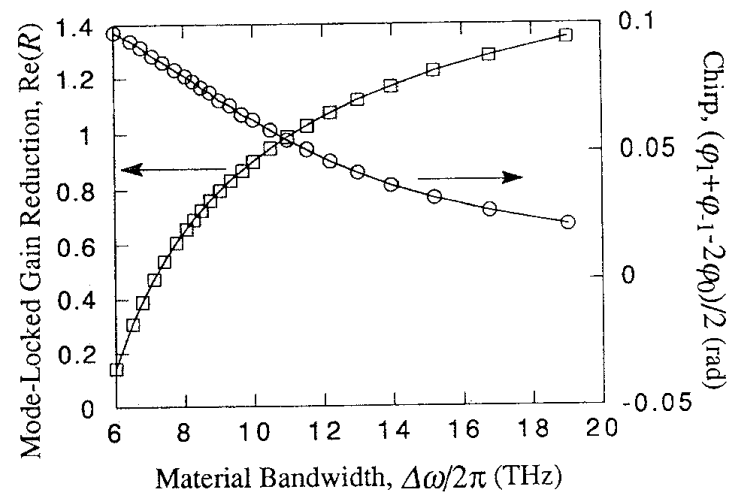

Fig. 18. Calculated value of $\operatorname{Re}(R)$, the reduction in threshold gain due to mode coupling, plotted against $\Delta \omega$, material bandwidth.

quency is expected to result in reduced mode coupling due to a reduction in $\kappa_{g}$ and $\kappa_{a}$. This will eventually lead to a point where the minimum mode coupling cannot be obtained and no stable mode-locked supermode exists. Although the point is $\approx 105 \mathrm{GHz}$ in this case (Fig. 17), using larger values of $s$ $(\approx 5)$, we have obtained stable supermode solutions slightly beyond $200 \mathrm{GHz}$. This agrees well with the theoretical results presented by Lau [8]. In this case, larger mode coupling effects resulted in a reduced downchirp.

As intuitively expected, lasers having a larger gain and absorber bandwidth will obtain a greater mode-locked gain reduction. Fig. 18 shows the expected increase in $\operatorname{Re}(R)$ as one solves the supermode equations allowing successively larger material bandwidths. Even larger advantages are found to occur if one assumes a gain bandwidth wider than the laser's absorption bandwidth. An expected decreased pulse chirp for larger material bandwidth is also shown in Fig. 18.

\section{EXPERIMENTAL RESULTS AND DISCUSSION}

Experimental measurements of the spectrum, pulse chirp, and the variation of pulse chirp with injection current have previously been published [20]. The laser used was a monolithic two-section quadruple quantum well GaAs laser having a repetition rate of $73 \mathrm{GHz}$. It showed qualitatively the same

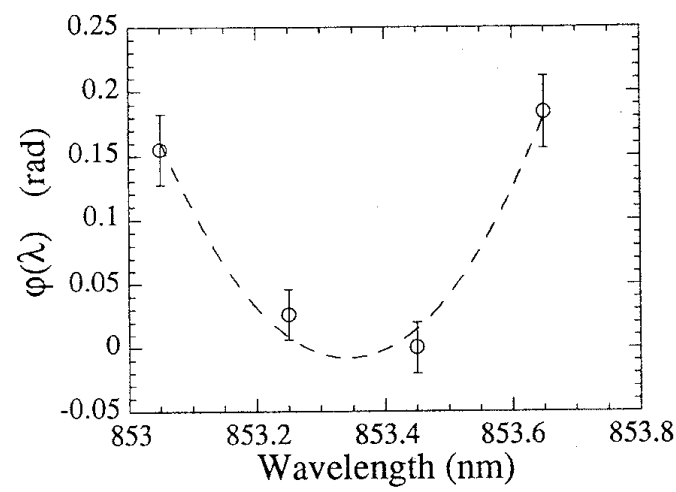

Fig. 19. Chirp measured in frequency domain, displayed in terms of the phase of optical spectrum versus the center wavelength of a $0.5 \mathrm{~nm}$ rectangular spectral filter. The measurement is done with $30 \mathrm{~mA}$ into gain section and with absorber grounded and shows an essentially linear downchirp of $1.7 \mathrm{ps} / \mathrm{nm}$.

characteristics as the calculation for $80 \mathrm{GHz}$ in the previous section. A broader spectrum and longer pulses as found from streak camera results can typically be obtained at higher bias conditions. An optical spectrum for the laser operating at $30 \mathrm{~mA}$ has previously been shown in [20]. The chirp of this spectrum has been measured through cross-correlation techniques [20], and integration of these results leads to phase values, $\phi(\lambda)$, of the optical spectrum plotted in Fig. 19. The figure shows a phase of the optical spectrum corresponding to a train of pulses with a $1.7 \mathrm{ps} / \mathrm{nm}$ downchirp and a timebandwidth product, $\Delta \tau \Delta \nu$, which is $18 \%$ larger than the compressed pulse time-bandwidth product achieved in the experiment. This regime of operation qualitatively corresponds to the calculated optical phase in Fig. 10. Additionally, the experimental measurement of $1.7 \mathrm{ps} / \mathrm{nm}$ downchirp for this laser is equivalent to $\left(\phi_{1}+\phi_{-1}-2 \phi_{0}\right) / 2=0.07 \mathrm{rad}$. Previously presented experimental results have demonstrated the effect of changes in the dc gain section injection current from the preceding case. The experimental results are shown in Fig. 20. While the laser is above threshold, the changes in dc injection current are nearly linearly related to the average photon intensity inside the cavity, $\tilde{s}_{0}$. Hence, we expect Fig. 20 to show agreement with the calculated pulse chirp in Fig. 12. Both show a sequence of upchirped, chirp-free, and downchirped operation as the photon intensity inside the cavity is increased.

Although good agreement between theory and experiment is obtained, we do not intend to imply that we have found the actual parameters of the mode-locked laser. However, we believe that the chosen parameters place the calculation in qualitatively the same regime of operation and that the calculated effects of the $\alpha$ parameter, the laser structure parameters, and the bias parameters will show a good correspondence with further experimental results.

Additionally, all results presented in this paper are believed to be for the lowest-order supermode - the one which possesses a minimum threshold gain. We have found some relatively small regions in the parameter space in which a second supermode solution could be found as a self-consistent solution. For a set of reasonable parameters and an arbitrary $\tilde{s}_{0}$, 


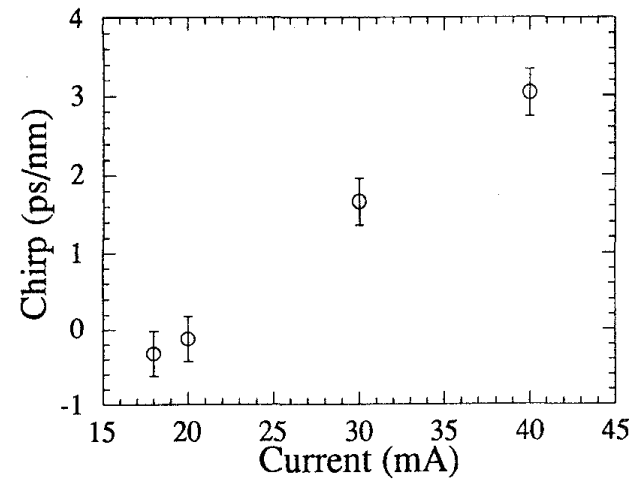

Fig. 20. Measured values of chirp as a function of gain section currents. An increasing downchirp is seen as the gain current is increased.

the second supermode was always found to exist for a slightly different repetition rate detuning, $\delta$, and a higher required gain, $\tilde{g}_{0}$. The supermode solutions were not orthogonal. This is contrary to some assumptions in a recent publication on passively mode-locked laser noise [22]. One would expect actively mode-locked supermode solutions to be orthogonal. However, passively mode-locked lasers are inherently nonlinear, i.e., mode coupling is a direct consequence of the saturation effects resulting from the beating of the Fabry-Perot laser modes. Only in a linear coupled mode problem would one expect the eigenvectors to be orthogonal. In passive mode-locking, however, the presence of one supermode will modify the system (the laser) such that conditions will not permit a second supermode to exist simultaneously. Thus a superposition of supermodes is not a valid solution to the set of coupled nonlinear equations. The characteristic shape of the second supermode we have found has essentially the same shape for its supermode envelope but was offset by half of one mode spacing from the usual spectrum center. Two of the lasing modes in its supermode thus possessed nearly equal field strengths, the nearly quadratic phase was essentially centered about this offset point and the necessary gain was always found to be higher than that required for the supermode which was not offset.

\section{CONCLUSION}

In conclusion, we presented a steady-state analysis for highrepetition-rate passively mode-locked semiconductor lasers. We derived an equation for an arbitrary mode that exists in the supermode of the laser. The equation requires gain to balance loss and incorporates phase effects that result from amplitude-to-phase coupling in each section of the laser. Additionally, mode coupling enters through the nonlinearity of both the saturable gain and saturable absorption sections. A nonlinear eigenvalue problem approach was presented to numerically solve for the passively mode-locked laser's supermode. An approximation of nearest-neighbor-only coupling was used in this paper. Next, an approximate three-mode solution was analytically solved for the purpose of building intuition and theoretically explaining recent experimental results which show the possibility of obtaining upchirped, chirp-free and downchirped pulses all from a single laser under different gain section bias. Results of the full supermode calculation (with nearest-neighbor-only coupling) were presented. The supermode magnitude and phase were plotted in the case where no amplitude-to-phase coupling exists in either laser section. Here, supermode solutions could be obtained over a broad range of cavity intensities. In this case, parameter values and mode-locking ranges show good agreement with previous calculations by Lau [8]. Other characteristics of the supermode solution were plotted as a function of cavity intensity also.

When reasonable amplitude-to-phase coupling factors were chosen for both laser sections, the supermode symmetry was severely changed. The phase was found to take on a predominantly quadratic shape in the region of the spectrum where the mode strengths are significant. This indicated the presence of essentially linearly chirped pulses. The presence of a nonzero $\alpha$ parameter was found to drastically limit the range (in terms the variation of cavity intensity) over which stable modelocked solutions could be found owing to the added phase effects. Near regions where the phase effects from gain and absorber nearly compensated each other, the effect of the $\alpha$ parameters on the reduction in gain due to coupling were neither very advantageous nor very harmful. They typically led to a slight weakening in $\operatorname{Re}(R)$, the reduction in threshold gain due to mode coupling. To facilitate understanding and optimization of high-repetition-rate passively mode-locked lasers, calculations of the reduction in gain provided through mode coupling and of the expected linear chirp were presented for variations in parameters of the laser structure and bias. Comparisons were made to expectations and to results from other models.

Next, experimental results from a high-repetition-rate passively mode-locked laser at $73 \mathrm{GHz}$ were compared to the supermode calculations in this paper. A good qualitative agreement for the spectral shape, chirp, and variation in chirp with changing injection current was found. The calculated supermodes analyzed were typically not as broad as the measured supermode. The reason for choosing narrower supermodes is that in this case the higher-order coupling effects (e.g., second nearest neighbor, third nearest neighbor coupling, etc.) are expected to be smaller. Thus, in this case, the nearest-neighborcoupling approximation is expected to be more accurate. However, by including second-nearest-neighbor coupling and higher-order coupling in the matrix for the supermode solution, one may more accurately model lower repetition rate modelocked lasers down to lower repetition rates $(\approx 5 \mathrm{GHz})$ which are viable for data rates in communication systems which are practical today.

\section{REFERENCES}

[1] H. A. Haus, "Theory of mode locking with a slow saturable absorber," IEEE J. Quantum Electron, vol. QE-11, pp. 736-746, Sept. 1975.

[2] O. E. Martinez, R. L. Fork, and J. P. Gordon, "Theory of passively mode-locked lasers for the case of a nonlinear complex-propagation coefficient," J. Opt. Soc. Amer. B, vol. 2, pp. 753-760, May 1985.

[3] J. C. Chen, H. A. Haus, and E. P. Ippen, "Stability of lasers modelocked by 2 saturable absorbers," IEEE J. Quantum Electron., vol. 29 , pp. 1228-1232, Apr. 1993.

[4] O. P. McDuff and S. E. Harris, "Nonlinear theory of the internally loss-modulated laser," IEEE J. Quantum Electron., vol. 3, pp. 101-111, Mar. 1967. 
[5] A. E. Siegman, Lasers. Mill Valley, CA: Univ. Sci., 1986, ch. 24.

[6] M. Sargent, M. O. Scully, and W. E. Lamb, Laser Physics. Reading. MA: Addison-Wesley, 1974, ch. 8.

[7] H. A. Haus, "A theory of forced mode-locking," IEEE J. Quantum Electron., vol. 11, pp. 323-329, July 1975.

[8] K. Y. Lau, "Narrow-band modulation of semiconductor lasers at millimeter wave frequencies $(>100 \mathrm{GHz}$ ) by mode locking," IEEE $J$ Quantum Electron., vol. 26, pp. 250-261, Feb. 1990.

[9] P. P. Vasil'ev and A. B. Sergeev, "Generation of bandwidth-limited 2 ps pulses with $100 \mathrm{GHz}$ repetition rate from multisegmented injection laser," Electron. Lett., vol. 25, pp. 1049-1050, Aug. 1989.

[10] S. Sanders, L. Eng, J. Paslaski, and A. Yariv, " $108 \mathrm{GHz}$ passive mode locking of a multiple quantum well semiconductor laser with an intracavity absorber," Appl. Phys. Lett., vol. 56, pp. 310-311, Jan. 1990.

[11] S. Arahira, S. Oshiba, Y. Matsui, T. Kunii, and Y. Ogawa, "500 GHz optical short pulse generation from a monolithic passively mode-locked distributed Bragg reflection laser diode," Appl. Phys. Lett., vol. 64, pp. 1917-1919, Apr. 1994

[12] Y. K. Chen and M. C. Wu, "Monolithic colliding-pulse quantum-well lasers," IEEE J. Quantum Electron., vol. 28, pp. 2176-2185, Oct. 1992

[13] J. F. Martins-Filho, E. A. Avrutin, C. N. Ironside, and J. S. Roberts, "Monolithic multiple colliding pulse mode-locked quantum-well lasers: Experiment and theory," IEEE J. Select. Topics Quantum Electron., vol. 1, pp. 539-551, June 1995 .

[14] J. H. Zarrabi, E. L. Portnoi, and A. V. Chelnokov, "Passive mode locking of a multiple quantum well semiconductor laser with an intracavity saturable absorber," Appl. Phys. Lett., vol. 59, pp. 1526-1528, Sept. 1991.

[15] K. Vahala, L. C. Chiu, S. Margalit, and A. Yariv, "On linewidth enhancement factor $\alpha$ in semiconductor injection lasers," Appl. Phys. Lett., vol. 42, pp. 631-633, Apr. 1983.

[16] A. Dienes, J. P. Heritage, M. Y. Hong, and Y. H. Chang, "Time-domain and spectral-domain evolution of subpicosecond pulses in semiconductor optical amplifiers," Opt. Lett., vol. 17, pp. 1602-1604, Nov. 1992.

[17] J. Paslaski and K. Y. Lau, "Parameter ranges for ultrahigh frequency mode locking of semiconductor lasers," Appl. Phys. Lett., vol. 59, pp. 7-9, July 1991

[18] V. Petrov and T. Mirtchev, "Chirping effects accompanying light pulse shortening by nonlinear amplification and saturable absorption," Optical Ouantum Electron., vol. 23, pp. 1161-1168, Dec. 1991.

[19] G. P. Agrawal and N. A. Olsson, "Self-phase modulation and spectral broadening of optical pulses in semiconductor laser amplifiers," IEEE J. Quantum Electron., vol. 25, pp. 2297-2306, Nov. 1989

[20] R. A. Salvatore and A. Yariv, "Demonstration of down-chirped and chirp-free pulses from high-repetition-rate passively mode-locked lasers," IEEE Photon. Technol. Lett., vol. 7, pp. 1151-1153, Oct. 1995

[21] S. Sanders, "Passive mode-locking and millimeter-wave modulation of quantum well lasers," Ph.D. dissertation, California Inst. of Technol., 1991.

[22] I. Kim and K. Y. Lau, "Frequency and timing stability of mode-locked semiconductor lasers-Passive and active mode locking up to millimeter wave frequencies," IEEE J. Quantum Electron., vol. 29, pp. 1081-1090, Apr. 1993.

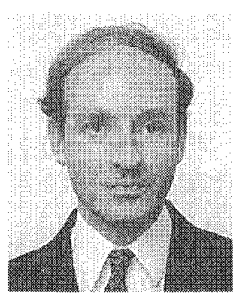

Randal A. Salvatore was born in Dearborn, MI, on January 2, 1967. He received the B.S. (summa cum laude) from the University of Michigan, Ann Arbor, in 1990, and the M.S. and Ph.D. from the California Institute of Technology in 1991 and 1995 respectively, all in electrical engineering.

He is presently an Assistant Research Engineer at the University of California, Santa Barbara. His current research interests are in semiconductor lasers, pulse shaping, and devices and systems for optical communications.

Dr. Salvatore is a member of the Optical Society of America, IEEE/LEOS Phi Beta Kappa, and Tau Beta Pi.

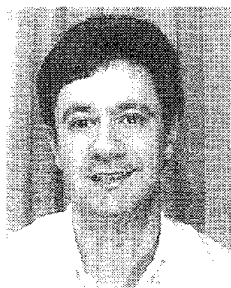

Steve Sanders was born in New York, NY on Apr. 18, 1964. He received the B.S. degree in physies and applied physics from Yale University in 1986, and the M.S. and Ph.D. degrees in applied physics from the California Institute of Technology, Pasadena, CA, in 1988 and 1991, respectively.

His graduate work was supported by a National Science Foundation Graduate Fellowship. His doctoral research involved the development of passively mode-locked quantum well lasers, resulting in the demonstration of monolithic devices emitting pulse trains at greater than $100 \mathrm{GHz}$ repetition rates. After completing his $\mathrm{Ph} . \mathrm{D}$, , he worked as a Research Fellow at the California Institute of Technology until 1993, on rare-earth doped fiber laser noise reduction. In 1993, he joined the research and development group at SDL. Inc. where he has been working on phasing of laser diode arrays to develop high power spatially coherent sources, and on application of high power spatially coherent laser diodes to frequency conversion for the generation of visible and mid-IR radiation.

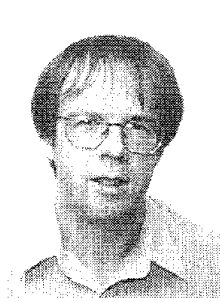

Thomas Schrans was born in Gent, Belgium, in 1964. He received the engineering degree in Electronics Engineering from the University of Gent, Belgium, in 1987. He received the M.S. and Ph.D. degrees from the California Institute of Technology in 1988 , and 1994

His doctoral research was on modelocking of semiconductor lasers, and modeling of DFB lasers. In 1987, he joined the California Institute of Technology on a one year fellowship from the Belgian American Educational Foundation. In 1994, he joined the IBM T. J. Watson Research Center, in Hawthorne, NY, as a Post-Doctoral Fellow, where he performed research on components and subsystems for WDM networks. Since June 1995, he is a Staff Scientist at Ortel Corporation, Alhambra, CA. His research interests are semiconductor lasers and optical communications.

Amnon Yariv (S'56-M'59-F'70-LF'95) was born in Tel Aviv, Israel. He received the B.S. (1954), M.S. (1956), and Ph.D. (1958) degrees in electrical engineering from the University of California in Berkeley.

In 1959, he went to Bell Telephone Laboratories, Murray Hill, NJ, joining the early stages of the laser effort. He came to the California Institute of Technology in 1964 as an Associate Professor of Electrical Engineering, and became a Professor in 1966. In 1980, he became the Thomas G Myers Professor of Electrical Engineering and Applied Physics. On the technical side, he took part (with various co-workers) in the discovery of a number of early solid-state laser systems, in proposing and demonstrating the field of semiconductor integrated optics, the invention of the semiconductor distributed feedback laser and in pioneering the field of phase conjugate optics. His present research efforts are in the areas of nonlinear optics, semiconductor lasers and integrated optics with emphasis on communication and computation. He is a founder and chairman of the board of the ORTEL Corporation. He has published widely in the laser and optics fields, and has written a number of basic texts in quantum electronics, optics and quantum mechanics.

Dr. Yariv is a member of the American Physical Society, Phi Beta Kappa, the American Academies of Arts and Sciences, the National Academies of Engineering and Sciences, and a Fellow of the Optical Society of America. He was the recipient of the 1980 Quantum Electronics Award of the IEEE, the 1985 University of Pennsylvania Pender Award, and the 1986 Optical Society of America Ives Medal, and the 1992 Harvey Prize. 